\title{
Portomesenteric and splenic vein thrombosis after laparoscopic sleeve gastrectomy
}

\author{
Saliha Karagöz Eren, (1) Olcay Çınar, (1) Alican Yalı, (1) Sedat Çarkıt, \\ (1) Ömer Topuz, (1) Tamer Ertan \\ Department of General Surgery, University of Health Sciences, Kayseri City Training and Research Hospital, Kayseri, Turkey
}

\begin{abstract}
Portomesenteric venous thrombosis (PVT) is a rare and life-threatening complication after laparoscopic bariatric surgery. Laparoscopic sleeve gastrectomy (LSG) is one of the most popular methods of bariatric surgical procedures. We aimed to present a 27 -year-old male patient who presented with thrombosis in the portal vein, superior mesenteric vein, and splenic vein following LSG. Patient presented with widespread abdominal pain in the epigastric region, loss of appetite, and constipation at the postoperative $41^{\text {st }}$ day; in color Doppler ultrasonography and computerized tomography, thrombosis was detected in portal vein and branches, splenic vein, portal confluence level, and superior mesenteric vein. The patient was given antithrombotic therapy and discharged without symptoms. The incidence of portal vein thrombosis has steadily increased with the increased number of laparoscopic bariatric operations. It requires a high degree of clinical suspicion as it presents with nonspecific symptoms. PVT should be considered in cases with atypical abdominal pain after bariatric surgery.

Keywords: Bariatric surgery; mesenteric vein thrombosis; obesity; portal vein thrombosis; sleeve gastrectomy; splenic vein thrombosis.
\end{abstract}

\section{Introduction}

Portal vein thrombosis was first reported in 1869 in a patient with liver cirrhosis. ${ }^{[1]}$ The American Association for the Study of Liver Diseases (AASLD) describes acute PVT as "the sudden formation of a thrombus in the portal vein". The thrombus may involve varying portions of the mesenteric and/or splenic veins, and PVT associated with emerging symptoms is often classified as acute. ${ }^{[2]}$ Chronic PVT occurs when a collateral hepatopetal vein network substitutes for the obstructed portal vein by bypassing the thrombosed parts of the vein. ${ }^{[2]}$ PVT is a life-threatening condition with several local and sys- temic etiologies. ${ }^{[3]}$ Laparoscopic surgical procedures with reported postoperative PVT include appendiceal and colon resections, cholecystectomy, splenectomy, hepatectomy, Nissen fundoplication for gastroesophageal reflux disease, and bariatric surgery for morbid obesity. [4,5] The increase in the incidence of PVT with the widespread use of laparoscopic techniques in general surgery over the last two decades suggests that PVT is not related to the direct manipulation of portal or large mesenteric vessels. Although the mechanism of PVT formation after laparoscopy is unknown, it has been suggested that the increase in intraabdominal pressure due to pneumoperi- 
toneum might have led to a decrease in portal venous blood flow, which in turn led to a relative prothrombotic state. ${ }^{[6,7]}$ Laparoscopic sleeve gastrectomy (LSG) is a popular single-stage operation in the treatment of morbid obesity with acceptable morbidity and long-term weight loss prospect compared to Roux-enY gastric bypass. ${ }^{[8]}$ Here we present the case of a 27 year-old male patient who had LSG and developed thrombosis in portal vein, superior mesenteric vein, and splenic vein on postoperative day 41 along with literature review.

\section{Case Report}

A 27-year-old male patient with a body mass index of 40.2 $\mathrm{kg} / \mathrm{m}^{2}$ had undergone LSG in accordance with the surgical guidelines after multidisciplinary preoperative preparations. ${ }^{[8]}$ A subcutaneous single dose of low molecular weight heparin (LMWH) (Clexane, $6000 \mathrm{IU}$, Sanofi) was applied for thromboembolic prophylaxis 12 hours before the operation. Immediately prior to the operation, an intermittent pneumatic compression device was fitted for deep vein thrombosis prophylaxis. After the induction of anesthesia, first-generation cephalosporin was intravenously (IV) administered to the patient.

The surgery was performed using a 5-port technique and under a $\mathrm{CO}_{2}$ pressure of $15 \mathrm{mmHg}$. The stomach was liberated with a large curvature bipolar vessel sealing system (LigaSure $^{\mathrm{TM}}$ ); sleeve gastrectomy was performed using Echelon stapler (Johnson and Johnson, New Brunswick, NJ) and SeamGuard staple line reinforcement (Gore, Flagstaff, AZ). A 36-Fr size endoluminal gastric tube was used to size the lumen and perform an intraoperative leak test, and at the end of the operation one Jackson-Pratt drain was placed along the stapler line. The general anesthesia procedure lasted 100 minutes. During posterior gastric dissection, the portomesenteric circulation was not visualized or manipulated.
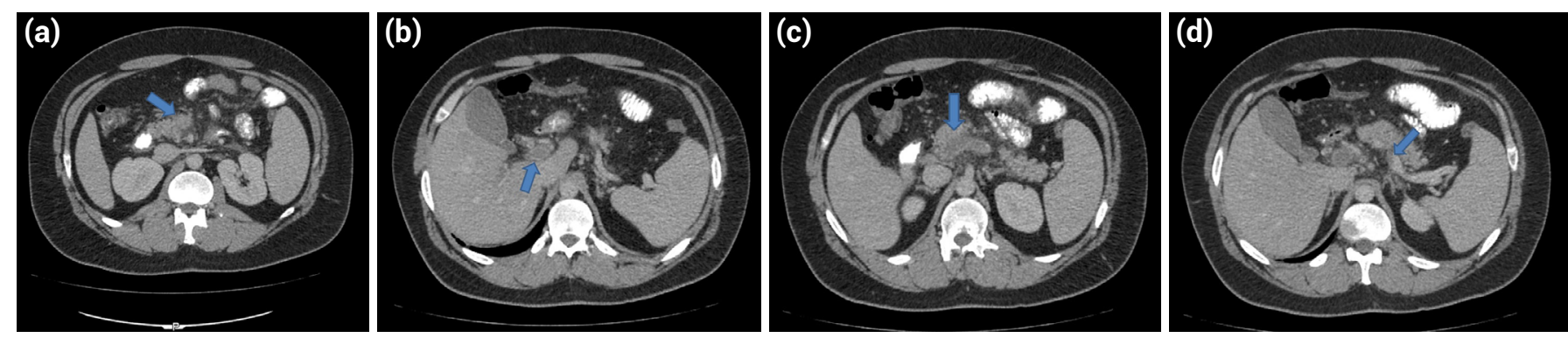

Figure 1. (a) Contrast-enhanced CT of the abdomen shows thrombosis in superior mesenteric vein (arrow), (b) Blue arrow indicates thrombosis of main portal vein (c) Blue arrow indicates thrombosis in portal confluence (d) Blue arrow indicates thrombosis of section of splenic vein.
The patient was mobilized at postoperative fourth hour, and at the $24^{\text {th }}$ hour the pneumatic compression device was removed and the patient wore compression socks. On the postoperative third day the patient was started on oral liquid food (low water). On the fourth day, the percutaneous drain was withdrawn and the patient was discharged with routine postoperative bariatric surgery recommendations and diet protocol. A single dose of subcutaneous LMWH 6000 IU was prescribed to be used daily during hospitalization and for at least 20 days after discharge.

The patient was rehospitalized on postoperative $41^{\text {st }}$ day with the complaints of abdominal pain, which was widespread but more prominent in the epigastric region, loss of appetite, constipation, and fatigue. Physical examination showed no other than minimal sensitivity in epigastric region, WBC: 9200/mm³ AST: 34 U/L ALT: 42 U/L and other routine biochemical parameters were normal. Abdominal ultrasonography (USG) was normal; thrombosis was detected in portal vein and branches, splenic vein, portal confluence level, and superior mesenteric vein in Doppler USG of the portal vein. In oral/IV contrast-enhanced abdominal and pelvic computerized tomography (CT), enlargement and filling defect compatible with acute thrombus were observed in portal vein, both branches of portal vein, superior mesenteric vein, and distal branches of superior mesenteric vein, extending to the proximal segment of splenic vein (Fig. 1a-d). No pathological thickening of the walls was observed in intestinal segments, neither intraabdominal free air nor significant acid (findings of ischemia or perforation). There was no evidence of any abscess in the abdomen or any finding to suggest a leak through the stapler line in the surgery site. Through consultation with gastroenterology, the patient was administered prophylactic antibiotherapy, oral warfarin (Coumadin, Eczacıbaşı-Baxter, $5 \mathrm{mg}$ ), and LMWH treatment (Clexane, 2x6000 IU, Sanofi). The patient's 
complaints improved in the 48-hour follow-up period; the patient was discharged with oral warfarin treatment to have an international normalized ratio (INR) of 2-3. In the follow-up three months later, the patient was found to follow the diet, exercise regularly, and have no clinical or laboratory problems.

In the follow-up Doppler USG of the portal vein in postoperative $6^{\text {th }}$ month, splenic venous diameter was normal and cavernous transformation, thrombus, or shunt was not observed in portal hilum.

\section{Discussion}

Portomesenteric venous thrombosis is a rare but potentially life-threatening condition after laparoscopic surgery. ${ }^{[4-6]}$ Postoperative PVT cases have become frequent with the widespread use of laparoscopic bariatric surgical procedures in recent years. Commonly reported complications of laparoscopic sleeve gastrectomy (LSG) include stapler line leak, respiratory failure, pulmonary embolism, hemorrhage, stricture, and splenic injury while the number of reported PVT cases is limited.

The mechanism of PVT formation after laparoscopic surgery has not been clearly elucidated. Portal venous flow is influenced by increased intraabdominal pressure due to carbon dioxide-induced pneumoperitoneum. Portosplenic venous flow is reduced by $>50 \%$ when $\mathrm{CO}_{2}$ pressure is above $14 \mathrm{mmHg} .{ }^{[9]}$ It is unclear how important this effect is in clinical terms but it is thought that reduced portal blood circulation may be associated with a tendency for thrombosis in the portomesenteric circulation. Besides, hypercapnia caused by $\mathrm{CO}_{2}$ insufflation may lead to decreased blood flow through vasoconstrictive activity. ${ }^{[9]}$ In addition, the reverse Trendelenburg position was reported to cause increased portal pressure, decreased portal blood flow, and additional stenosis in the portal venous system during laparoscopic bariatric surgery. ${ }^{[5,9]}$

Local surgical factors that cause venous thrombosis tendency are direct surgical manipulation of the portomesenteric vessels as in splenectomy. Therefore, PVT after laparoscopic splenectomy has been evaluated separately from other laparoscopic surgical procedures. ${ }^{[6]}$ The ligation of the splenic vein causes endothelial damage, which increases the thrombogenicity of this vessel. In sleeve gastrectomy, only short gastric veins are dissected, without direct dissections of portomesentlric vessels. In these cases, other etiologic factors should be evaluated well.
Obesity is associated with an increased thromboembolic risk; consequently, all bariatric surgeries carry an increased risk. ${ }^{[10]}$ Besides, acquired thrombophilia may contribute to PVT, especially in the bariatric population. The incidence of inherited thrombophilia reported in patients with post-bariatric PVT ranges from $3 \%$ to $43 \%{ }^{[5]}$ In patients who underwent bariatric surgery, the most commonly reported risk factors for PVT were heterozygous prothrombin 20210 mutation and protein $C$ and protein $\mathrm{S}$ deficiency, and less frequently factor $\mathrm{V}$ Leiden mutation, increased factor VIII level, elevated fibrinogen level, methylenetetrahydrofolate deficiency, JAK2 mutation and lupus anticoagulants. ${ }^{[5]}$ In our case, there were no family history to suggest a genetic coagulation disorder or no history of smoking and possible hematologic and genetic reasons were investigated in consultation with hematology department and no pathological finding was detected.

Although it is still a controversial topic, some studies suggest that the risk of thromboembolism following the bariatric surgery is extended after discharge from the hospital, and therefore the prophylaxis should be continued for several weeks postoperatively. ${ }^{[1]}$ However, reduction of PMVT risk through this approach is not theoretically accepted as the standard. ${ }^{[12]}$

Non-invasive imaging methods are adequate for the diagnosis of PMVT; more invasive methods such as portal venography are not usually necessary. Contrast-enhanced CT or color Doppler ultrasound may be suitable for diagnosis. ${ }^{[13]}$ In our case, the diagnosis was confirmed by both imaging modalities.

Current guidelines recommend starting anti-coagulant treatment after diagnosis; additional treatment should be given if there is a known underlying cause. ${ }^{[14]}$ The recommended treatment period is 6 to 12 months. In patients with a diagnosed systemic prothrombotic condition, a lifelong therapy may be required for the recanalization of portal vein. ${ }^{[15]}$ More aggressive treatment modalities such as endovascular thrombolysis or percutaneous thrombectomy are available to prevent permanent thrombosis or intestinal ischemia. ${ }^{[16]}$ There are no large studies supporting the routine use of thrombolytics but it has been shown to be effective in cases resistant to standard anticoagulation therapy. ${ }^{[17]}$ In addition, the risk of life-threatening bleeding should be considered in this treatment. In our case, the patient's symptoms dramatically improved with anticoagulant therapy and there was no need for thrombolytic therapy. 
In our patient, morbid obesity and having undergone laparoscopic surgery in the reverse Trendelenburg position were potential risk factors for postoperative PVT. As the prevalence of bariatric surgery continues to increase, the incidence of PVT may increase but PVT may become a treatable condition with early diagnosis. While bariatric surgery patients are often treated with routine pharmacological deep venous thrombosis (DVT) prophylaxis, its role in the prevention of PVT is not known yet.

\section{Conclusion}

Portal vein thrombosis is a complication that has potentially life-threatening consequences following laparoscopic bariatric surgery. It should be of clinical suspicion as it presents with nonspecific symptoms. In cases with nonspecific abdominal pain after bariatric surgery, possible PVT diagnosis should be kept in mind, and necessary radiological procedures should be used for early diagnosis and treatment.

\section{Disclosures}

Informed Consent: Written informed consent was obtained from the patient for the publication of the case report and the accompanying images.

Peer-review: Externally peer-reviewed.

Conflict of Interest: None declared.

\section{References}

1. Handa P, Crowther M, Douketis JD. Portal vein thrombosis: a clinician-oriented and practical review. Clin Appl Thromb Hemost 2014;20:498-506. [CrossRef]

2. DeLeve LD, Valla DC, Garcia-Tsao G; American Association for the Study Liver Diseases. Vascular disorders of the liver. Hepatology 2009;49:1729-64. [CrossRef]

3. Parikh S, Shah R, Kapoor P. Portal vein thrombosis. Am J Med 2010;123:111-9. [CrossRef]

4. James AW, Rabl C, Westphalen AC, Fogarty PF, Posselt AM, Campos GM. Portomesenteric venous thrombosis after laparoscopic surgery: a systematic literature review. Arch Surg 2009;144:520-6. [CrossRef]

5. Ikeda M, Sekimoto $M$, Takiguchi S, Kubota M, Ikenaga M, Yamamoto $\mathrm{H}$, et al. High incidence of thrombosis of the portal venous system after laparoscopic splenectomy: a prospective study with contrast-enhanced CT scan. Ann Surg 2005;241:208-16. [CrossRef]

6. Shoar S, Saber AA, Rubenstein R, Safari S, Brethauer SA, AlThani $\mathrm{H}$, et al. Portomesentric and splenic vein thrombosis
(PMSVT) after bariatric surgery: a systematic review of 110 patients. Surg Obes Relat Dis 2018;14:47-59. [CrossRef]

7. Jakimowicz J, Stultiëns G, Smulders F. Laparoscopic insufflation of the abdomen reduces portal venous flow. Surg Endosc 1998;12:129-32. [CrossRef]

8. Mechanick JI, Youdim A, Jones DB, Garvey WT, Hurley DL, McMahon MM, et al; American Association of Clinical Endocrinologists; Obesity Society; American Society for Metabolic \& Bariatric Surgery. Clinical practice guidelines for the perioperative nutritional, metabolic, and nonsurgical support of the bariatric surgery patient--2013 update: cosponsored by American Association of Clinical Endocrinologists, The Obesity Society, and American Society for Metabolic \& Bariatric Surgery. Obesity (Silver Spring) 2013;21 Suppl 1:S1-27. [CrossRef]

9. Rottenstreich A, Elazary R, Kalish Y. Abdominal thrombotic complications following bariatric surgery. Surg Obes Relat Dis 2017;13:78-84. [CrossRef]

10. Eichinger S, Hron G, Bialonczyk C, Hirschl M, Mimar E, Wagner 0 , et al. Overweight, obesity, and the risk of recurrent venous thromboembolism. Arch Intern Med 2008;168:1678-3.

11. Steele KE, Schweitzer MA, Prokopowicz G, Shore AD, Eaton $\mathrm{LC}$, Lidor $\mathrm{AO}$, eta al. The long-term risk of venous thromboembolism following bariatric surgery. Obes Surg 2011;21:13716. [CrossRef]

12. Clinical Issues Committee of the American Society for Metabolic and Bariatric Surgery. Prophylactic measures to reduce the risk of venous thromboembolism in bariatric surgery patients. Surg Obes Relat Dis 2007;3:494-5. [CrossRef]

13. Bradbury MS, Kavanagh PV, Bechtold RE, Chen My, Ott DJ, Regan JD, et al. Mesenteric venous thrombosis: diagnosis and noninvasive imaging. Radiographics 2002;22:527-41.

14. Sanyal AJ. Acute portal vein thrombosis in adults: clinical manifestations, diagnosis, and management. Available at: https://www.uptodate.com/contents/acute-portal-veinthrombosis-in-adults-clinical-manifestations-diagnosisand-management?search=\%20acute-portal-vein-thrombosis-in-adults-clinical-manifestations-diagnosis-\%20andmanagement. \&source = search_result \&select edTitle=1 150\&usage_type=default\&display_rank=1. Accessed Sep 9, 2018.

15. Condat B, Pessione F, Hillaire S, Denninger MH, Guillin MC Poliquin $\mathrm{M}$, et al. Current outcome of portal vein thrombosis in adults: risk and benefit of anticoagulant therapy. Gastroenterology 2001;120:490-7. [CrossRef]

16. Tateishi A, Mitsui H, Oki T, Morishita J, Maekawa H, Yahagi N, et al. Extensive mesenteric vein and portal vein thrombosis successfully treated by thrombolysis and anticoagulation. $J$ Gastroenterol Hepatol 2001;16:1429-33. [CrossRef]

17. Hollingshead M, Burke CT, Mauro MA, Weeks SM, Dixon RG, Jaques PF. Transcatheter thrombolytic therapy for acute mesenteric and portal vein thrombosis. J Vasc Interv Radiol. 2005;16:651-61. [CrossRef] 